\title{
Mutación del gen K-ras en tumores pancreático-biliares
}

\author{
Juan Carlos Roa $\mathbf{S}^{1}$, Leonardo Anabalón $\mathbf{R}^{\mathbf{1}}, \mathbf{0}$ scar Tapia $\mathbf{E}^{\mathbf{1}}$, \\ Angélica Melo $A^{1}$, Xabier de Aretxabala U ${ }^{2}$, Iván Roa $E^{1,3}$. \\ Frequency of $K$-ras mutation \\ in biliary and pancreatic tumors
}

Background: The ras gene family (H-ras, N-ras and K-ras) are oncogenes that mutate frequently in human cancer, specially in tumors of the biliary tract and pancreas. Aim: To determine the frequency of K-ras gene codon 12 mutation in pancreatic and biliary tumors. Material and Methods: Samples of 35 gallbladder, 15 ampulla of Vater, 10 biliary tract and 9 pancreatic tumors, were analyzed. The tumor tissue was microdissected from paraffin embedded biopsies. The mutation was detected by a combination of polymerase chain reaction (PCR) and restriction fragment length polymorphism (RFLP). Results: Overall, $46 \%$ of samples had K-ras gene mutations. Mutation frequency was 80, 56, 50 and 29\% for ampulla of Vater, pancreatic, biliary tract and gallbladder tumors, respectively. When compared with the rest, gallbladder tumors had a significantly lower frequency of the mutation. Median survival for biliary tract tumors was 6 months, compared with 65 months for gallbladder tumors ( $p<0.05$ ). Conclusions: Gallbladder carcinoma had the lower frequency of K-ras mutation, when compared with pancreatic, biliary tract and ampulla of Vater tumors (Rev Méd Chile 2005; 133: 1434-40).

(Key Words: Biliary tract neoplasms; Gallbladder neoplasms; Genes, ras; Pancreatic neoplasms)

Recibido el 16 de marzo, 2005. Aceptado el 4 de julio, 2005.

Trabajo financiado por Proyecto Fondecyt \#1010523 y \#1050603.

${ }^{1}$ Departamento de Anatomía Patológica, Facultad de Medicina, Universidad de la Frontera.

${ }^{2}$ Departamento de Cirugía, Clínica Alemana de Santiago. ${ }^{3}$ Departamento de Patología, Clí-

nica Alemana de Temuco.

L a genética molecular de los tumores del complejo pancreático biliar ha sido extensamente estudiada. Nuestra población presenta una alta incidencia de tumores de la vesícula biliar y menor frecuencia de tumores de páncreas, ampo-

Correspondencia a: Juan Carlos Roa S. Facultad de Medicina, Universidad de la Frontera. Temuco, Chile. Casilla 54D. E mail: jcroa@ufro.cl lla de Vater y de vías biliares, las razones de estas diferencias no están claras y requieren estudios adicionales ${ }^{1}$.

La asociación de mutaciones del gen K-ras con este tipo de tumores y lesiones preneoplásicas, específicamente adenomas, ha sido documentada ${ }^{2-4}$. Sin embargo, existen muy pocos estudios basados en nuestra población ${ }^{5,6}$.

El oncogén K-ras pertenece a la familia de genes ras (H-ras, N-ras y K-ras) ${ }^{7}$. Se ubica sobre el 
cromosoma 12p12 y tiene aproximadamente $45 \mathrm{~Kb}$ en extensión ${ }^{8}$. Las proteínas codificadas por estos genes adoptan un conformación estructural con un peso de $21 \mathrm{Kd}$, se localizan en la membrana plasmática y participan en la vía de transducción de señales y diferenciación celular ${ }^{5,7}$. La mutación de este gen es el evento genético más común de la carcinogénesis humana ${ }^{9}$. Más de $90 \%$ de las mutaciones se han encontrado en el codón 12 y con menor frecuencia en los codones 13 y $61^{10,11}$. Ha sido reportada en diferentes órganos con alta frecuencia en tumores de colon, $50 \% 12$, pulmón, $50 \% 13$ y tiroides, $60 \% 14$. En el cáncer de páncreas se han comunicado frecuencias de mutación, que varían entre 70 y $100 \%$ de los casos $^{8,9,15}$. En el cáncer ampular entre 19 y 70\%, para tumores de vía biliar ${ }^{16-22}$ entre 0 y $55 \% 20,23-27$ y entre 0 y 100\% para vesícula biliar ${ }^{6,20,23,25}$.

Existen diversos métodos para la detección de mutaciones de K-ras, sin embargo, uno de los más usados corresponde a una técnica combinada de PCR (reacción en cadena de la polimerasa) y RFLP utilizando enzimas de restricción (polimorfismo en la longitud de los fragmentos de restricción) $)^{5,9,28,29}$.

El objetivo de este trabajo fue determinar la frecuencia de mutación en el codón 12 del gen K-ras, mediante PCR-RFLP, en tumores pancreático-biliares en nuestra población y correlacionarla con las características clínicas y morfológicas de los tumores.

\section{MATERIAL y MÉTODOS}

Casos. Se seleccionaron 69 casos de cánceres pancreático-biliares: 35 de cáncer de vesícula biliar (CVB), 15 de ampolla de Vater (AV), 10 de vía biliar (BTC) y 9 de cáncer pancreático (CP) de los archivos del Servicio de Anatomía Patológica del Hospital Hernán Henríquez Aravena y del Departamento de Patología de la Universidad de la Frontera.

Extracción del ADN. Se utilizó tejido fijado en formalina e incluido en parafina. Se microdisecaron manualmente 5 cortes histológicos con áreas representativas del tumor. El ADN fue extraído con el Kit Puregene DNA Isolation System (Gentra
Systems, USA), de acuerdo a las instrucciones del fabricante. El $\mathrm{ADN}$ fue almacenado a $-20^{\circ} \mathrm{C}$ hasta su análisis. En todos los casos se realizó amplificación del gen B-globina para confirmar la integridad y calidad del ADN.

Detección de mutación del codón 12 del gen K-ras. Se utilizó un PCR en seminido y RFLP, con iniciadores previamente reportados en la literatu$\mathrm{ra}^{5}$. Estos iniciadores (primers) permiten amplificar un fragmento del gen K-ras que incluye un sitio de restricción para la enzima MvaI. Tanto los productos PCR (pPCR) de la primera como de la segunda amplificación, fueron digeridos con la enzima MvaI (10 U para 20 ul pPCR) ${ }^{9,28,29}$. El iniciador K-ras-A tiene una base cambiada con respecto a la secuencia del gen K-ras. Esto produce un sitio de restricción para la enzima MvaI cuando el codón 12 es normal. También el iniciador K-ras-B tiene una base cambiada, lo que lo hace susceptible de digestión por la enzima MvaI y de esta manera no interfiere en la segunda amplificación.

Los pPCR de la segunda amplificación fueron corridos en un gel al 10\% de poliacrilamida (1:29), Buffer TBE 1X, Glicerol 5\% a 150 Volt, por $2 \mathrm{~h}$ y posteriormente teñidos con bromuro de etidio (Figura 1).

Controles. Como control positivo, se empleó ADN de carcinomas de páncreas en los que previamente se había demostrado la mutación del K-Ras en el codón 12. Como control negativo se usó ADN genómico comercial (Promega) y como control blanco se sustituyó el ADN por agua deionizada.

Estadística. La relación entre la mutación de K-ras con variables categóricas tales como sexo, raza, tipo histológico, se estableció usando la prueba de chi cuadrado o la prueba exacta de Fisher. Para variables numéricas como edad se utilizó la prueba de $\mathrm{t}$ de Student. Para el cálculo de sobrevida global, se incluyó sólo a los casos avanzados, la asociación con parámetros morfológicos y clínicos y la presencia de mutación se realizó mediante múltiples curvas de Kaplan Meier y fueron comparadas utilizando la prueba de logrank y el modelo de Cox Hazard. 


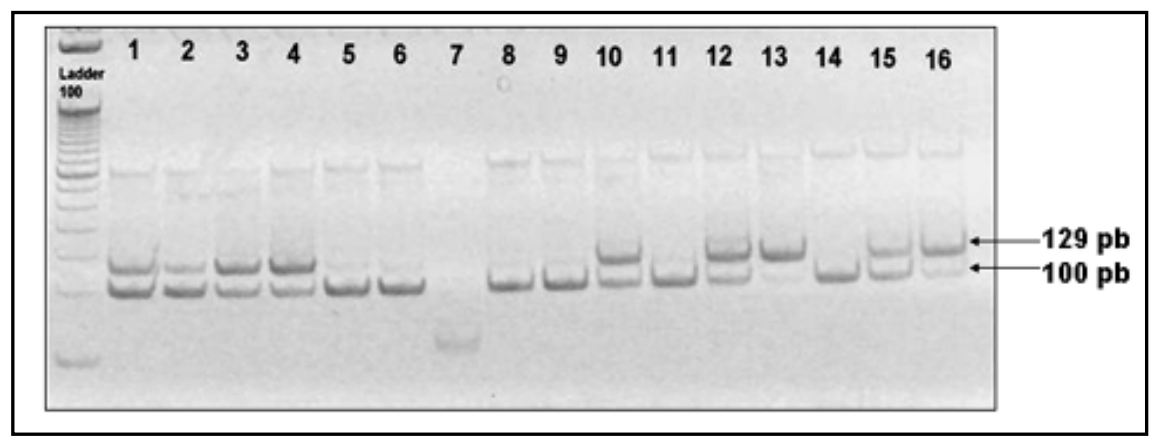

Figura 1. Mutaciones del codón 12 del gen K-ras en tumores pancreático-biliares. Gel de poliacrilamida al 10\% teñido con bromuro de etidio. En el cual se observan los productos del método PCR-RFLP. El producto mutado es de $129 \mathrm{pb}$. La presencia de 2 bandas se explica por la presencia de ADN mutado y normal simultáneamente en la muestra analizada. (Cáncer de Ampolla de Vater Líneas 1-3 vesícula biliar líneas 4-6, vía biliar líneas 8-12, cáncer de páncreas líneas 13-15. Las líneas 7 y 16 corresponden al blanco y control positivo, respectivamente.

\section{RESULTADOS}

Las características generales del grupo de pacientes incluidos en el estudio, se detallan en la Tabla 1. La mayoría de los casos de carcinoma de vesícula biliar correspondieron a mujeres (89\%), en los otros tumores, la relación fue similar 0 levemente superior para el sexo masculino (40\%56\%). Las muestras de tumores estudiados correspondieron a pacientes no mapuches (71\%). El rango de edad fluctuó entre los 25 y 92 años, con un promedio de 57,6 años.

El tipo histológico predominante correspondió al tubular (83\%). El grado de infiltración de los tumores estudiados eran mayoritariamente tumores avanzados (95,7\%) y moderadamente diferenciados (57\%). La totalidad de CP y AV fueron intervenidos mediante pancreatoduodenectomía más disección ganglionar, 12 de los 17 casos de tumores vesiculares subserosos recibieron tratamiento quirúrgico complementario posterior a la colecistectomía, mediante bisegmentectomía hepática y disección ganglionar. Ninguno de los pacientes con tumores de vía biliar fue sometido a resección quirúrgica con intención curativa. Se encontraron diferencias significativas en la sobrevida global según el origen del tumor, siendo el cáncer de vía biliar y el de vesícula biliar los de peor y mejor pronóstico, respectivamente $(p=0,004)$ (Tabla 1). También se observó diferencias en la sobrevida según tipo histológico de los tumores (peor pronóstico para adenocarcinomas tubulares) $(p<0,05)$ y según grado de diferenciación histológica (mejor pronóstico para tumores bien diferenciados) ( $\mathrm{p}<0,05$ ).

En la totalidad de los casos fue posible amplificar adecuadamente los productos de PCR requeridos (Figura 1). En 32 de los 69 casos $(46,4 \%)$ se detectó la mutación en el codón 12 del gen K-ras (Tabla 1). Según el órgano analizado, las frecuencias de mutación fueron de $29 \%, 50 \%$, $56 \%$ y $80 \%$, para CVB, BTC, CP y AV, respectivamente. Cuando agrupamos a los tumores pancreático-biliares no vesiculares y los comparamos con el CVB, la frecuencia de mutación fue de $64,7 \%$ y $35,3 \%$, respectivamente (Tabla 2$)(p<0,0001)$. El grupo de pacientes que presentaba tumores de ampolla de Vater con mutación del gen K-ras tuvo mejor sobrevida $(p=0,05)$. Se observó una tendencia no significativa a mayor frecuencia de mutación en hombres $(p=0,07)$ (Tabla 1).

\section{DisCUSIÓN}

La asociación de mutaciones del gen K-ras con este tipo de tumores y lesiones preneoplásicas, específicamente adenomas, ha sido bien documentada. Sin embargo, existen muy pocos estudios basados en nuestra población.

Nuestros resultados muestran que aproximadamente la mitad de los tumores pancreático-biliares, presentan mutación en el codón 12 del gen K-ras (46,4\%). La frecuencia de mutación fue dependiente del tipo de tumor $(p<0,05)$, estando 
Tabla 1. Características generales del grupo analizado y su relación con sobrevida y mutación del gen k-ras $(n=69)$

\begin{tabular}{|c|c|c|c|c|c|c|c|c|}
\hline & Variable & Vesícula biliar & Páncreas & Ampolla & Vía Biliar & Total & $\begin{array}{c}\text { Mutación de K-rras } \\
\text { del total (\%) }\end{array}$ & \\
\hline $\mathrm{n}^{0}$ de casos & & 35 & 9 & 15 & 10 & 69 & 69 & \\
\hline \multirow[t]{2}{*}{ Sexo } & Masculino & $11 \%(4)$ & $56 \%(5)$ & $53 \%$ (8) & $40 \%(4)$ & $21(30 \%)$ & $61,9 \%(13 / 21)$ & $\mathrm{p}=0,07(\mathrm{~A})$ \\
\hline & Femenino & $89 \%(31)$ & $44 \%$ (4) & $47 \%(7)$ & $60 \%(6)$ & $48(70 \%)$ & $39,5 \%$ (19/48) & \\
\hline \multirow[t]{2}{*}{ Etnia } & Mapuche & $34 \%$ (12) & $11 \%$ (1) & $20 \%(3)$ & $40 \%$ (4) & $20(29 \%)$ & $45 \%(9 / 20)$ & $p=n s$ \\
\hline & no Mapuche & $66 \%(23)$ & $89 \%$ (8) & $80 \%$ (12) & $60 \%(6)$ & $49(71 \%)$ & $46,9 \%$ (23/49) & \\
\hline \multirow[t]{2}{*}{ Edad } & Promedio & 56,8 años & 54,4 años & 56,2 años & 65,4 años & 57,6 años & & $\mathrm{p}=\mathrm{ns}$ \\
\hline & Rango & 32-92 años & $25-70$ años & 41-69 años & $45-83$ años & 25-92 años & & \\
\hline \multirow[t]{2}{*}{ Sobrevida } & Promedio & 65,5 meses & 25,2 meses & 18,5 meses & 6,1 meses & 41 meses & & $\mathrm{p}=0,004$ (B) \\
\hline & Rango & 0-194 meses & 1-88 meses & $0-54$ meses & 1-11 meses & 0-194 meses & & \\
\hline \multirow[t]{5}{*}{ Tipo histológico } & Tubular & 80\% (28) & $78 \%$ (7) & 80\% (12) & $100 \%(10)$ & $83 \%(57)$ & $46 \%(26 / 57)$ & $\mathrm{p} \varangle 0,05$ (C) \\
\hline & Tubulo-papilar & $11 \%(4)$ & & $20 \%(3)$ & & $10 \%(7)$ & $71 \%(5 / 7)$ & \\
\hline & Mucinoso & $6 \%(2)$ & & & & $2,9 \%(2)$ & $0 \%(0 / 2)$ & \\
\hline & Escamoso & $3 \%(1)$ & $11 \%(1)$ & & & $2,9 \%(2)$ & $50 \%(1 / 2)$ & \\
\hline & Indiferenciado & & $11 \%(1)$ & & & $1,4 \%(1)$ & $0 \%(0 / 1)$ & \\
\hline \multirow[t]{2}{*}{ Etapa } & Incipiente & & & $20 \%(3)$ & & $4,3 \%$ (3) & $67 \%(2 / 3)$ & $p \varangle 0,05$ (D) \\
\hline & Avanzado & $100 \%$ (35) & $100 \%(9)$ & $80 \%$ (12) & $100 \%(10)$ & $95,7 \%(66)$ & $33 \%(29 / 66)$ & \\
\hline \multirow[t]{3}{*}{ Grado de diferenciación } & Bien & $9 \%$ (3) & & $33 \%(5)$ & & $11,6 \%(8)$ & $75 \%(6 / 8)$ & $p \varangle 0,05$ (E) \\
\hline & Moderado & $54 \%(19)$ & $56 \%(5)$ & $60 \%(9)$ & $60 \%(6)$ & $56,5 \%(39)$ & $41 \%(16 / 39)$ & \\
\hline & Poco & $37 \%$ (13) & $44 \%$ (4) & $7 \%$ (1) & $40 \%$ (4) & $31,9 \%(22)$ & $45 \%(10 / 22)$ & \\
\hline \multirow[t]{2}{*}{ Mutación K-ras } & Presente & $29 \%(10)$ & $56 \%(5)$ & $80 \%$ (12) & $50 \%(5)$ & $46,4 \%(32)$ & & $p \varangle 0,05$ (F) \\
\hline & Ausente & $71 \%(25)$ & $44 \%$ (4) & $20 \%(3)$ & $50 \%(5)$ & $53,6 \%(37)$ & & \\
\hline
\end{tabular}

A: Mutación de K-ras según sexo. B: Sobrevida según localización del tumor. C: Sobrevida según tipo histológico. D: Sobrevida según etapa. E: Sobrevida según grado de diferenciación. F: Mutación de K-ras según localización del tumor.

Tabla 2. M utación de K-ras en tumores vesiculares vs tumores no vesiculares

\begin{tabular}{|lccc|}
\hline & $\begin{array}{c}\text { Tumores } \\
\text { vesiculares }\end{array}$ & $\begin{array}{c}\text { Tumores no } \\
\text { vesiculares }\end{array}$ & Total \\
\hline K-ras mutado & 10 & 22 & 32 \\
K-ras no mutado & 25 & 12 & 37 \\
Total & 35 & 44 & 69 \\
\hline
\end{tabular}

$\mathrm{P}<0,0001$.

presente en $29 \%$ de los adenocarcinomas de la vesícula biliar, en $56 \%$ y $50 \%$ para carcinomas de páncreas y vía biliar respectivamente y en $80 \%$ de los carcinomas de la ampolla de Vater.

En la vesícula biliar se han reportado frecuencias de mutación de K-ras que fluctúan entre 0 y $100 \% 4,30$. Algunos autores han postulado que aquellos tumores que se asocian a una unión bilio-pancreática anómala, condición más frecuente en las series japonesas, presentarían mayor frecuencia de mutación de K-ras ${ }^{31,32}$. Sin embargo, otras series han encontrado que la frecuencia de esta mutación es comparable con series de pacientes que no muestran esta anormali$\operatorname{dad}^{30,33,34}$. En nuestros casos se encontró una frecuencia de $29 \%$ de mutación, muy similar a una serie previamente reportada ${ }^{5}$. Esto contrasta con el 2,4\% de mutaciones reportadas en una serie de 73 pacientes chinos de CVB $^{4,30}$, en los que $70 \%$ se encontraban asociados a litiasis. En nuestra región, más de $90 \%$ de los cánceres vesiculares se encuentran asociados a litiasis ${ }^{1}$, lo cual podría explicar algunas de las diferencias genético-moleculares encontradas en diferentes poblaciones y áreas geográficas.

En el cáncer de páncreas se han publicado frecuencias de mutación de K-ras que fluctúan entre 70 y $100 \% 15$, sin diferencias significativas para 
los distintos estadios de la enfermedad ${ }^{18}$. Parece ser que esta mutación estaría involucrada en la iniciación, pero no en la progresión, del cáncer pancreático. En nuestros 9 casos encontramos una frecuencia algo menor de mutación que alcanzó a $56 \%$ de los casos. Sin embargo, las diferencias encontradas no fueron significativas. No encontramos relación con características morfológicas 0 sobrevida con la presencia de mutación.

En los tumores de vía biliar se han reportado frecuencias de mutación que varían entre 14,3\% y $55 \%$ 4,30,35,36 y para el cáncer de ampolla entre 19 y $70 \% 16,17$. En nuestra serie encontramos frecuencias de mutación de K-ras de 50 y 80\% para tumores de vía biliar y de ampolla de Vater, respectivamente.

Las marcadas diferencias reportadas en las frecuencias de mutación de K-ras pueden ser atribuibles al uso de diferentes técnicas 0 a diferencias raciales y geográficas. Ejemplo de esto es la alta incidencia de mutación de K-ras, en colangiocarcinomas, observada en Tailandia y Japón ${ }^{37}$.

Nuestros casos no mostraron relación entre la mutación de K-ras y las características morfológicas del tumor. En la literatura se ha reportado escasa asociación con características morfológicas. Howe et al comunicaron que la mutación se asociaba a tumores de ampolla mayores de $2 \mathrm{~cm}$, sin embargo, no se encontró relación con sobrevida ${ }^{22}$.

En nuestro estudio, la mutación de K-ras en cáncer de ampolla de Vater resultó ser un factor de mejor pronóstico, contrariamente a lo previamente reportado 20,26 , sin embargo, esta observación se encuentra basada en una muestra limitada. En los tumores pancreático-biliares estudiados, la mutación de K-ras no mostró valor pronóstico al igual que otras comunicaciones 22,26 , sin embargo, otros autores han encontrado que la presencia de K-ras se encuentra asociada a peor pronóstico ${ }^{30,35}$.

\section{REFERENCIAS}

1. Roa I, Araya JC, de Aretyabala X, Salinas C, Wistuba I. Patología de la vesícula biliar en Temuco, IX región. Rev Méd Chile 1989; 117: 889-94.

2. Wistuba II, Albores-SaAvedRa J. Genetic abnormalities involved in the pathogenesis of gallbladder carcinoma. J Hepatobiliary Pancreat Surg 1999; 6: 237-44.
Nuestro estudio encontró diferencias significativas en la sobrevida, dependiendo del órgano comprometido y también de características clínicas y morfológicas tales como sexo, tipo histológico y grado de diferenciación $(p<0,05)$. Aun cuando sólo se incluyeron tumores avanzados en este análisis, no todos los pacientes fueron sometidos a resecciones quirúrgicas con intención curativa. La imposibilidad de estratificar a los diferentes tipos de tumores debido al tamaño de la muestra limita su análisis.

Concluimos que la frecuencia de mutación del codón 12 del gen K-ras en tumores biliopancreáticos es similar a la reportada en otras áreas geográficas y en otras etnias. Aun cuando nuestra serie de tumores pancreático-biliares sólo mostró tener impacto pronóstico en los cánceres de ampolla y estar asociado más frecuente a la población femenina, se corrobora la importancia de este gen, que se encuentra mutado en casi $50 \%$ de todos los casos estudiados.

De esta manera, podemos señalar que la mutación del codón 12 del gen K-ras es un evento que se observa en forma frecuente en los tumores pancreático-biliares de nuestra población. Al igual que en series internacionales reportadas, existe una significativa diferencia entre la frecuencia de mutación entre los cánceres vesiculares y los no vesiculares, lo cual sugiere que a pesar de ser tumores anatómicamente relacionados, tienen vías carcinogenéticas distintas, por lo cual las estrategias para su estudio a nivel genético-molecular deberán ser distintas.

El mejor entendimiento de la biología molecular de los tumores pancreático-biliares no es solo de interés en investigación básica sino que llegará a tener implicancia clínica en la evaluación de riesgo de desarrollar neoplasias, en el diagnóstico precoz, tratamiento y pronóstico.

3. Wistuba II, Miquel JF, Gazdar AF, Albores-Saavedra J. Gallbladder adenomas have molecular abnormalities different from those present in gallbladder carcinomas. Hum Pathol 1999; 30: 21-5.

4. RASHID A. Cellular and molecular biology of biliary tract cancers. Surg Oncol Clin N Am 2002; 11: 995-1009.

5. Roa JC, Roa I, de Aretxabala X, Melo a, Faría G, TAPIA O. Mutación del gen K-ras en el cáncer 
de la vesícula biliar. Rev Méd Chile 2004; 132: 955-60.

6. Wistuba II, Sugio K, Hung J, Kishimoto Y, Virmani AK, RoA I ET AL. Allele-specific mutations involved in the pathogenesis of endemic gallbladder carcinoma in Chile. Cancer Res 1995; 55: 2511-5.

7. WALSH AB, BAR-SAGI D. Differential activation of the Rac pathway by Ha-Ras and K-Ras. J Biol Chem 2001; 276: 15609-15. Epub 2001 Feb 14.

8. Mu DQ, Peng YS, Xu QJ. Values of mutations of K-ras oncogene at codon 12 in detection of pancreatic cancer: 15 years experience. World J Gastroenterol 2004; 10: 471-5.

9. REN YX, Xu GM, L ZS, Song YG. Detection of point mutation in K-ras oncogene at codon 12 in pancreatic diseases. World J Gastroenterol 2004; 10: 881-4.

10. Yoshida T, OhNami S, Aoki K. Development of gene therapy to target pancreatic cancer. Cancer Sci 2004; 95: 283-9.

11. CERNY WL, Mangold KA, SCARPe山 DG. K-ras mutation is an early event in pancreatic duct carcinogenesis in the Syrian golden hamster. Cancer Res 1992; 52: 4507-13.

12. VogelsTein KKB. Colorectal tumors. In: Bert V, editor. The genetic basis of human cancer. Second ed. New York: Mac Graw Hill; 2002; 583612.

13. Clements NC Jr, Nelson MA, Wymer JA, Savage C, Aguirre M, Garewal H. Analysis of K-ras gene mutations in malignant and nonmalignant endobronchial tissue obtained by fiberoptic bronchoscopy. Am J Respir Crit Care Med 1995; 152: 1374-8.

14. Esapa CT, Johnson SJ, KendaL-Taylor P, Lennard TW, HaRRIS PE. Prevalence of Ras mutations in thyroid neoplasia. Clin Endocrinol (Oxf) 1999; 50: 529-35.

15. Sakorafas GH, Tsiotou AG, Tsiotos GG. Molecular biology of pancreatic cancer; oncogenes, tumour suppressor genes, growth factors, and their receptors from a clinical perspective. Cancer Treat Rev 2000; 26: 29-52.

16. Matsubayashi $H$, Watanabe $H$, Yamaguchi $T$, Ajioka Y, NishiKURA K, KiJIMA H ET AL. Differences in mucus and K-ras mutation in relation to phenotypes of tumors of the papilla of Vater. Cancer 1999; 86: 596-607.

17. Zhao B, Kimura W, Futakawa N, Muto T, Kubota K, HaRIHARA Y ET AL. p53 and p21/Waf1 protein expression and $\mathrm{K}$-ras codon 12 mutation in carcinoma of the papilla of Vater. Am J Gastroenterol 1999; 94: 2128-34.

18. Motojima K, Tsunoda T, Kanematsu T, Nagata $Y$, URANo T, ShiKu H. Distinguishing pancreatic carcinoma from other periampullary carcinomas by analysis of mutations in the Kirsten-ras oncogene. Ann Surg 1991; 214: 657-62.

19. Scarpa A, Cape山 P, Zamboni G, Oda T, Mukai $K$, BonetTI F ET AL. Neoplasia of the ampulla of Vater. Ki-ras and p53 mutations. Am J Pathol 1993; 142: 1163-72.

20. Malats N, Porta M, Corominas JM, Pinol JL, Rifa J, REAL FX. Ki-ras mutations in exocrine pancreatic cancer: association with clinico-pathological characteristics and with tobacco and alcohol consumption. PANK-ras I Project Investigators. Int J Cancer 1997; 70: 661-7.

21. Chung CH, Wilentz RE, Polak MM, Ramsoekh TB, Noorduyn LA, Gouma DJ ET AL. Clinical significance of K-ras oncogene activation in ampullary neoplasms. J Clin Pathol 1996; 49: 460-4.

22. Howe JR, Kumstra DS, Cordon-Cardo C, Paty PB, PARK PY, BrenNan MF. K-ras mutation in adenomas and carcinomas of the ampulla of vater. Clin Cancer Res 1997; 3: 129-33.

23. Tada M, Yokosuka O, Omata M, Ohto M, Isono K. Analysis of ras gene mutations in biliary and pancreatic tumors by polymerase chain reaction and direct sequencing. Cancer 1990; 66: 930-5.

24. Levi S, Urbano-Ispizua A, GiL R, Thomas DM, Gilbertson J, Foster C et al. Multiple K-ras codon 12 mutations in cholangiocarcinomas demonstrated with a sensitive polymerase chain reaction technique. Cancer Res 1991; 51: 3497-502.

25. Imai M, Hoshi T, Ogawa K. K-ras codon 12 mutations in biliary tract tumors detected by polymerase chain reaction denaturing gradient gel electrophoresis. Cancer 1994; 73: 2727-33.

26. Rujken AM, van Gulik TM, Polak MM, Sturm PD, Gouma DJ, OfFerhaus GJ. Diagnostic and prognostic value of incidence of K-ras codon 12 mutations in resected distal bile duct carcinoma. J Surg Oncol 1998; 68: 187-92.

27. Suto T, Habano W, Sugai T, Uesugi N, Funato O, KanNo S ET aL. Aberrations of the K-ras, p53, and APC genes in extrahepatic bile duct cancer. J Surg Oncol 2000; 73: 158-63.

28. Zhou M, Huang SG, Wan HY, Li B, Deng WW, L M. Genetic polymorphism in matrix metallopro- 
teinase- 9 and the susceptibility to chronic obstructive pulmonary disease in Han population of south China. Chin Med J (Engl) 2004; 117: 1481-4.

29. Uemura T, Hibi $K$, Kaneko T, Takeda S, Inoue $S$, Окосні O Ет AL. Detection of K-ras mutations in the plasma DNA of pancreatic cancer patients. J Gastroenterol 2004; 39: 56-60.

30. Rashid A, Ueki T, Gao YT, Houlhan PS, Waldace C, WANG BS ET AL. K-ras mutation, p53 overexpression, and microsatellite instability in biliary tract cancers: a population-based study in China. Clin Cancer Res 2002; 8: 3156-63.

31. Hanada K, Itoh M, Fuji $\mathrm{K}$, Tsuchida A, Ooishi $\mathrm{H}$, KajIYAMA G. K-ras and p53 mutations in stage I gallbladder carcinoma with an anomalous junction of the pancreaticobiliary duct. Cancer 1996; 77: 452-8.

32. Hidaka E, Yanagisana A, Seki M, Takano K, SetoguCHI T, Kato Y. High frequency of K-ras mutations in biliary duct carcinomas of cases with a long common channel in the papilla of Vater. Cancer Res 2000; 60: 522-4.
33. Kim SW, Her KH, Jang JY, Kim WH, Kim YT, Park $\mathrm{YH}$. K-ras oncogene mutation in cancer and precancerous lesions of the gallbladder. J Surg Oncol 2000; 75: 246-51.

34. Ajiki T, Fujimori T, Onoyama H, Yamamoto M, Kitazawa S, MAEDA S ET AL K-ras gene mutation in gallbladder carcinomas and dysplasia. Gut 1996; 38: 426-9.

35. Ahrendt SA, Rashid A, Chow JT, Eisenberger CF, PitT HA, SidRansKy D. p53 overexpression and Kras gene mutations in primary sclerosing cholangitis-associated biliary tract cancer. J Hepatobiliary Pancreat Surg 2000; 7: 426-31.

36. Watanabe $\mathrm{H}, \mathrm{Ha} A, \mathrm{Hu} Y \mathrm{X}$, Ohtsubo K, Yamaguchi Y, Мотоо Y Eт AL. K-ras mutations in duodenal aspirate without secretin stimulation for screening of pancreatic and biliary tract carcinoma. Cancer 1999; 86: 1441-8.

37. Kiba T, Tsuda H, Pairojkul C, Inoue S, Sugimura T, HIROHASH S. Mutations of the p53 tumor suppressor gene and the ras gene family in intrahepatic cholangiocellular carcinomas in Japan and Thailand. Mol Carcinog 1993; 8: 312-8. 